\title{
Analytical approach to chromatic correction in the final focus system of circular colliders
}

\author{
Yunhai Cai \\ SLAC National Accelerator Laboratory, 2575 Sand Hill Road, Menlo Park, California 94205, USA
}

(Received 21 January 2016; published 28 November 2016)

\begin{abstract}
A conventional final focus system in particle accelerators is systematically analyzed. We find simple relations between the parameters of two focus modules in the final telescope. Using the relations, we derive the chromatic Courant-Snyder parameters for the telescope. The parameters are scaled approximately according to $\left(L^{*} / \beta_{y}^{*}\right) \delta$, where $L^{*}$ is the distance from the interaction point to the first quadrupole, $\beta_{y}^{*}$ the vertical beta function at the interaction point, and $\delta$ the relative momentum deviation. Most importantly, we show how to compensate its chromaticity order by order in $\delta$ by a traditional correction module flanked by an asymmetric pair of harmonic multipoles. The method enables a circular Higgs collider with $2 \%$ momentum aperture and illuminates a path forward to $4 \%$ in the future.
\end{abstract}

DOI: 10.1103/PhysRevAccelBeams.19.111006

\section{INTRODUCTION}

In $e^{+} e^{-}$colliders, it has been always challenging to focus the beam to extremely small size at the collision point while maintaining an adequate momentum bandwidth. In the Bfactories [1,2], $1 \%$ of their momentum aperture was sufficient to accommodate the energy spread of the beam due to synchrotron radiation. For a circular Higgs factory (CHF) $[3,4]$ at $120 \mathrm{GeV}$ beam energy, the required momentum acceptance has to be larger than $2 \%$ to accommodate the beamstrahlung [5]. Moreover, the beam has to be focused even more to achieve the factory-level luminosity since the beam current is limited by the synchrotron radiation power [6]. The combination of extreme focus at the interaction point (IP) and large momentum aperture in the collider presents a grand challenge in the development of a CHF. In this paper, we will systematically investigate the chromatic effects in the final focus system and lay the ground work for the design of such colliders.

\section{PARAXIAL OPTICS}

The locations of the focusing point relative to its principle plane illustrated in Fig. 1 characterize a conventional optical system. Here, we will apply these well-known properties to the optics of charged particles in the transverse dimensions, namely the horizontal or vertical plane.

Given a 2-by-2 R-matrix of the optical system, we can show

Published by the American Physical Society under the terms of the Creative Commons Attribution 4.0 International license. Further distribution of this work must maintain attribution to the author(s) and the published article's title, journal citation, and DOI.

$$
\begin{aligned}
& p=\frac{R_{22}}{R_{21}}, \\
& q=-\frac{R_{11}}{R_{21}}, \\
& f=-\frac{1}{R_{21}},
\end{aligned}
$$

where $R_{11}, R_{21}$, and $R_{22}$ are the elements of the $\mathrm{R}$-matrix. The derivation requires the symplecticity of the R-matrix and validity of the paraxial approximation, $\tan \theta \approx \theta$, where $\theta$ is the angle of optical ray.

Inversely, given these geometrical parameters in the optical system, we can rewrite its R-matrix as

$$
R=\left(\begin{array}{cc}
\frac{q}{f} & \frac{1}{f}\left(p q+f^{2}\right) \\
-\frac{1}{f} & -\frac{p}{f}
\end{array}\right) .
$$

\section{DOUBLET}

As an example, we consider a doublet drawn schematically in Fig. 2. Its R-matrix can be obtained by multiplying the three R-matrices of the elements. Then applying Eq. (1), we have its geometrical parameters,

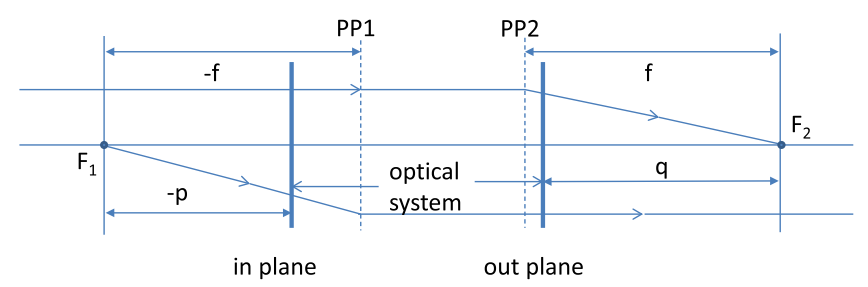

FIG. 1. Focusing points and geometrical parameters in a conventional optical system. 


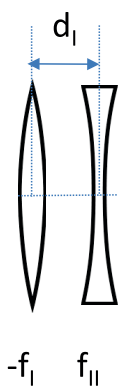

FIG. 2. A schematic layout of a doublet.

$$
\begin{aligned}
& p=-\frac{f_{I}\left(f_{I I}+d_{I}\right)}{d_{I}-f_{I}+f_{I I}}, \\
& q=\frac{f_{I I}\left(f_{I}-d_{I}\right)}{d_{I}-f_{I}+f_{I I}}, \\
& f=\frac{f_{I} f_{I I}}{d_{I}-f_{I}+f_{I I}} .
\end{aligned}
$$

Here we have to use the thin lens calculation for the quadrupoles. Sometimes it is useful to have its inverse,

$$
\begin{aligned}
f_{I} & =\frac{f^{2}+p q}{f-q}, \\
f_{I I} & =-\frac{f^{2}+p q}{f+q}, \\
d_{I} & =\frac{f^{2}+p q}{f} .
\end{aligned}
$$

To focus the charged particles in both transverse planes simultaneously, a doublet is often required because a magnetic quadrupole focuses in one plane while defocuses in the other. We tabulate the parameters of a final focus doublet used in this paper in Table I. The negative sign of $p_{1}$ means that the first focus point is on the left side of the in plane.

\section{TELESCOPE}

With focusing modules such as doublets, we consider how to use them to construct a telescope, which is a pointto-point and parallel-to-parallel imaging system and can be defined by its R-matrix,

TABLE I. Parameters of the final focus doublet.

\begin{tabular}{ll}
\hline \hline Physical parameters & Optical parameters \\
\hline$f_{I}: 1.36174 \mathrm{~m}$ & $p_{1}:-1.97756 \mathrm{~m}$ \\
$f_{I I}: 2.51748 \mathrm{~m}$ & $q_{1}:-0.41273 \mathrm{~m}$ \\
$d_{I}: 1.85541 \mathrm{~m}$ & $f_{1}: 1.13848 \mathrm{~m}$ \\
\hline \hline
\end{tabular}

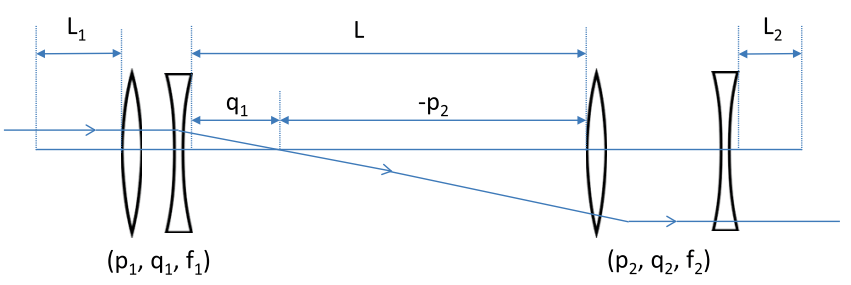

FIG. 3. A schematic layout of a telescope system that consists of two focusing modules.

$$
R=\left(\begin{array}{cc}
-M & 0 \\
0 & -1 / M
\end{array}\right)
$$

Here, $M$ is the demagnification factor. It was first introduced to particle accelerators by Karl Brown [7] when he designed the final focus system in the Stanford Linear Collider. He found many good chromatic properties in the system. In particular, he also provided a one-dimensional analysis, which unfortunately defocused the beam in the other plane and therefore cannot be used for charged particles. Here we will extend his work to a general telescope system, shown in Fig. 3, which can provide focus to the charged particles in both planes.

Given the optical parametrizations of R-matrices in the form of Eq. (2) for the two focusing modules, we can show that the parameters for the second module satisfy

$$
\begin{aligned}
& p_{2}=q_{1}-L, \\
& q_{2}=L_{2}+M^{2}\left(L_{1}+p_{1}\right), \\
& f_{2}=M f_{1},
\end{aligned}
$$

in order to make a telescope. The first condition has a geometrical interpretation as illustrated in Fig. 3. This simple picture has provided us the intuition to introduce the optical parameters in the final telescope. Moreover, it makes the system from the entry of the first module to the exit of the second one a parallel-to-parallel imaging system. Two drifts on flanks restricted by the second condition then makes the whole system a point-to-point imaging system as well. The third condition is merely a byproduct of this two-step procedure.

Since we had not used the doublets in the derivation, the focus module does not have to be a doublet as shown in Fig. 3. For the telescopes in particle accelerators, the conditions in Eq. (6) should be satisfied both horizontally and vertically with a different demagnification factor. If the thin-lens doublets are used, we can derive the focus lengths and the distance between the lens of the second doublet in terms of the first ones by applying Eqs. (3), (6), and (4).

\section{CHROMATIC OPTICS}

It is well known [8] that the dynamics of a charged particle in accelerators can be described by tracking its 


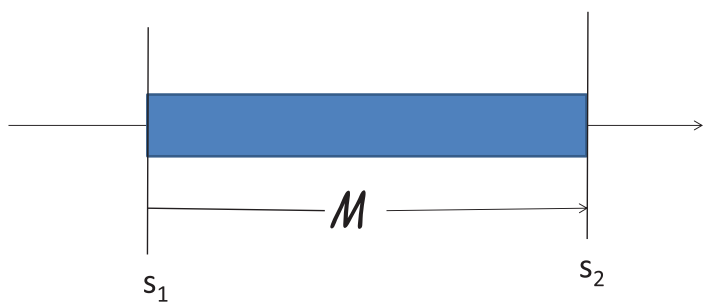

FIG. 4. An element in the beam line represented by its transfer map of the canonical coordinates $z=\left(x, p_{x}, y, p_{y}, \delta, \ell\right)$ from position $s_{1}$ to $s_{2}$.

canonical coordinates $z=\left(x, p_{x}, y, p_{y}, \delta, \ell\right)$, where $\delta$ is the relative momentum deviation and $\ell=v t$; $v$ is the velocity of the particle, and $t$ is the time of flight. For a magnetic element schematically illustrated in Fig. 4, its transfer map $\mathcal{M}$ can be obtained by solving the Hamilton's equations.

Under the paraxial approximation, the transfer map of a drift can be written as

$$
\begin{aligned}
& \mathcal{M}_{3}=y+\frac{L_{D}}{1+\delta} p_{y}, \\
& \mathcal{M}_{4}=p_{y}
\end{aligned}
$$

where $L_{D}$ is the length for the drift. Its $\delta$ dependence introduces the chromatic effects into the charged particle optics in the formulation of the canonical system. How to use the transfer maps to compute the chromatic optics order by order in $\delta$ has been studied [9]. It is worth noting that Eq. (7) is known as " chromatic drift" and often used in the beam-beam simulations [10,11].

Given the explicit matrices in Eq. (2) of the two focus modules and the transfer maps of the draft, a transfer map of the telescope can be obtained by concatenation of the maps, starting from the identity map. Up to the second order, we have

$$
\begin{aligned}
\mathcal{M}_{3}= & -M y+\frac{M L\left(L_{1}+p_{1}\right)}{f_{1}^{2}} y \delta \\
& +\frac{f_{1}^{2}\left(L_{2}+M^{2} L_{1}\right)+M^{2} L\left(L_{1}+p_{1}\right)^{2}}{M f_{1}^{2}} p_{y} \delta, \\
\mathcal{M}_{4}= & -\frac{1}{M} p_{y}-\frac{L}{M f_{1}^{2}} y \delta-\frac{L\left(L_{1}+p_{1}\right)}{M f_{1}^{2}} p_{y} \delta .
\end{aligned}
$$

A similar expression is applicable in the horizontal plane. Here we write out the vertical component because its demagnification factor is much larger than the horizontal one in electron accelerators. Its linear part simply confirms the validity of Eq. (6). The map can be simplified to

$$
\begin{aligned}
\mathcal{M}_{3} & =-M y+M L^{*} p_{y} \delta, \\
\mathcal{M}_{4} & =-\frac{1}{M} p_{y},
\end{aligned}
$$

if we assume, $M \gg 1$ and $L_{1}+p_{1} \approx 0$, which implies that the first focus point of the first module is extremely close to the interaction point in the vertical plane. Moreover, we have used $L^{*}=L_{1}$ to be consistent with the standard notation for the parameters near the IP. In our numerical example shown in Fig. 5, using the value of $p_{1}$ in Table I, it easy to see that this approximation is excellent.

The simplified map in Eq. (9) can be used to propagate [9] the Courant-Snyder parameters [12] through the final telescope. We find

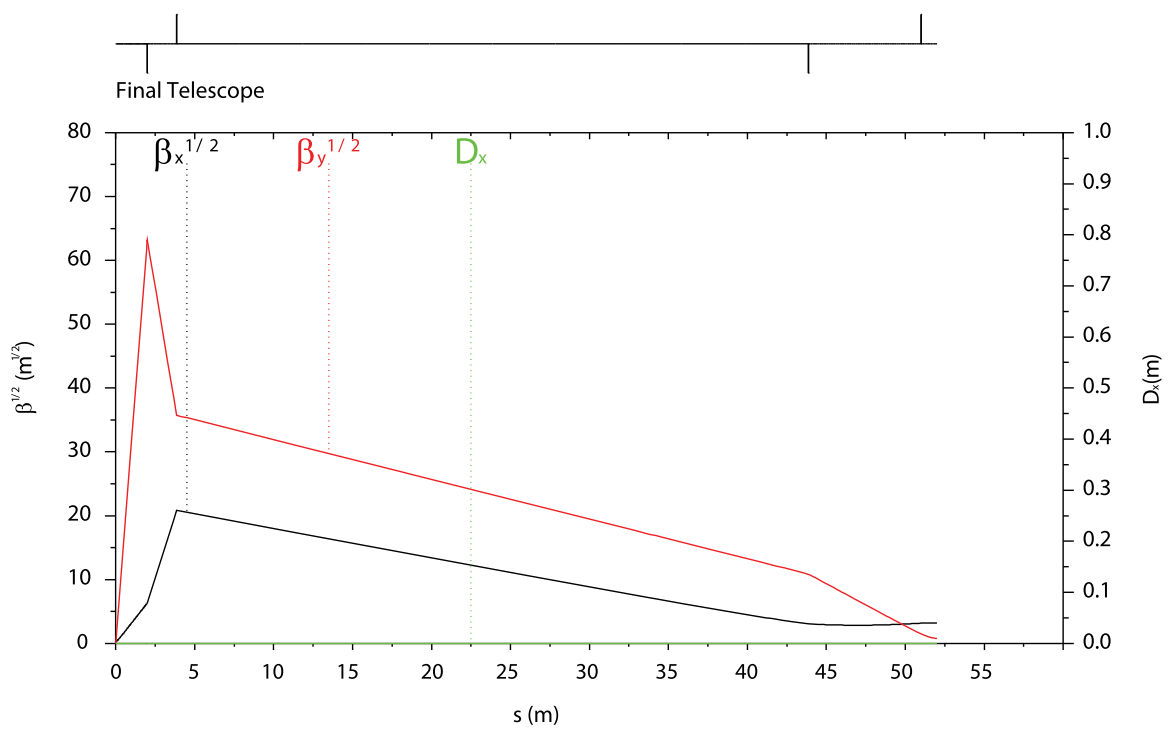

FIG. 5. The Courant-Synder parameters in a final telescope with demagnification factors $M: 10$ and 25 in the horizontal and vertical planes respectively. The parameters are $L^{*}=2 \mathrm{~m}, L=40 \mathrm{~m}, L_{2}=1 \mathrm{~m}, \beta_{x}^{*}=0.1 \mathrm{~m}$, and $\beta_{y}^{*}=1 \mathrm{~mm}$. 

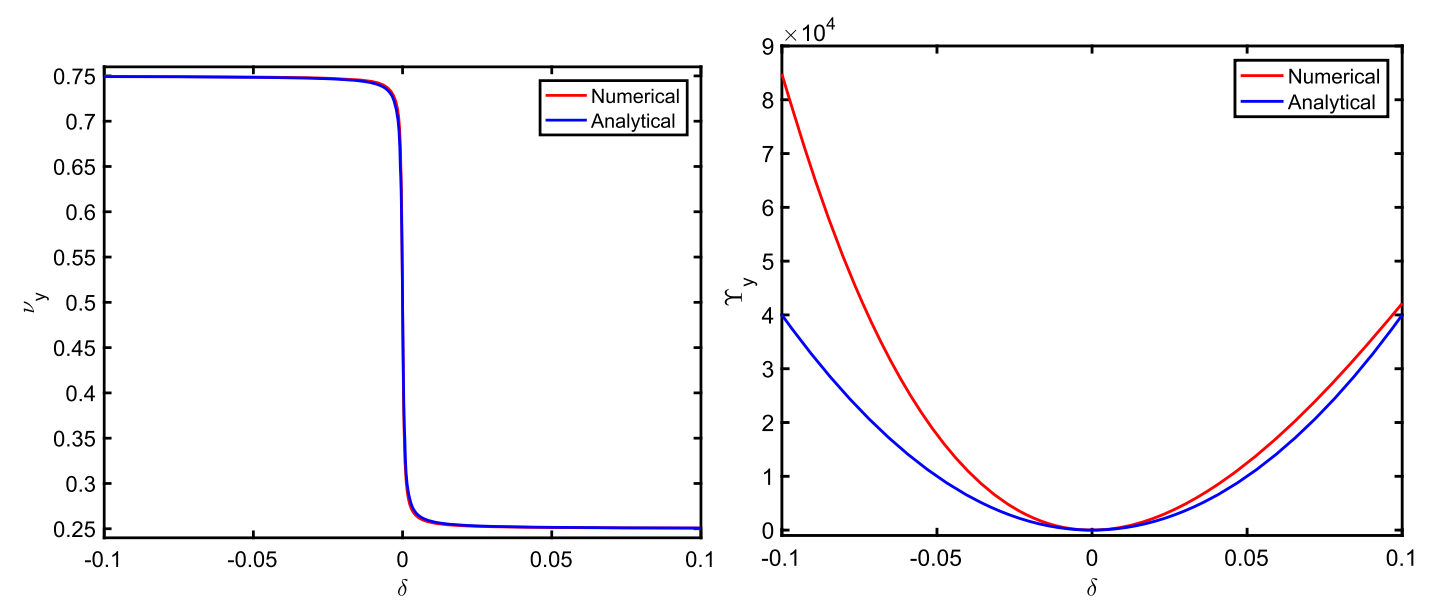

FIG. 6. (Left) The vertical phase advance in unit of $2 \pi$, namely $\nu_{y}=\psi_{y} / 2 \pi$ and (right) the Sands' mismatch parameter $\Upsilon$ as a function of $\delta$.

$$
\begin{aligned}
& \beta_{y}(\delta)=M^{2} \beta_{y}^{*}\left[1+\left(\frac{L^{*}}{\beta_{y}^{*}} \delta\right)^{2}\right], \\
& \alpha_{y}(\delta)=\frac{L^{*}}{\beta_{y}^{*}} \delta \\
& \psi_{y}(\delta)=\pi-\tan ^{-1}\left(\frac{L^{*}}{\beta_{y}^{*}} \delta\right) .
\end{aligned}
$$

Here we have assumed $\alpha_{y}^{*}=0$ at the IP. It is worth noting that the chromatic effects are scaled according to $\left(L^{*} / \beta_{y}^{*}\right) \delta$ for the final telescope with a very large demagnification factor. For the future $e^{+} e^{-}$colliders, the parameter $\left(L^{*} / \beta_{y}^{*}\right)$ is very large and at the order of a few thousands. Unfortunately, it is mostly determined by particle physicists and there is not much that can be done by accelerator experts.

Using the example shown in Fig. 5, the vertical phase advance in units of $2 \pi$ is computed numerically and plotted at the left in Fig. 6. In a comparison the last expression in Eq. (10). $\nu_{y}(\delta)$ varies in a large range of tune space: $0.5 \pm 0.25$. The tune change must be reduced to nearly zero within a reasonable bandwidth of $\delta$ if it is to be used in circular colliders.

Aside from the tune change, we need to have a good measure of the chromatic matching. We first consider a well-known parameter called $B_{\text {mag }}$ [13] that is commonly used to measure the mismatch between the beam distribution and the machine optics. Here we introduce it to describe the mismatch between chromatic optics to the machine optics designed for the on-momentum particle. Naturally, we have

$$
B_{\mathrm{mag}}(\delta)=\frac{1}{2}\left[\gamma_{0} \beta(\delta)-2 \alpha_{0} \alpha(\delta)+\beta_{0} \gamma(\delta)\right],
$$

where $\alpha_{0}, \beta_{0}$, and $\gamma_{0}$ are the lattice functions with $\delta=0$. There is a slightly different mismatch parameter introduced by Sands,

$$
\Upsilon(\delta)=B_{\mathrm{mag}}(\delta)+\sqrt{B_{\mathrm{mag}}^{2}(\delta)-1}
$$

In this paper, we choose to use the Sands' one because of its geometrical interpretation [14]. Furthermore, we find $\Upsilon^{\prime}=W$, where $W$ is the amplitude of the Montague functions [15]. As a result, $\Upsilon(\delta)$ could be considered as an extension for describing the high-order chromaticity. It is plotted at the right in Fig. 6 for the example with a comparison between the numerical computation and analytical formula derived from Eq. (10). The huge quadratic chromaticity wall is clearly seen in the figure. It severely limits the bandwidth of the focus system and has to be corrected. It is worth noting that the importance of this quadratic term was first emphasized by Bogomyagkov and Levichev [16] from a viewpoint of the conventional perturbation theory.

\section{CHROMATIC CORRECTION}

If there are dispersions, the chromaticity can be corrected locally be placing sextupole near the quadrupoles. For the linear colliders, such a scheme was proposed [17], achieving a large bandwidth in the momentum deviation. In order to generate the dispersions at the final doublets, it required a nonzero dispersion slope at the interaction point. Unfortunately, this kind of configuration enhances the synchrobetatron resonances through the beam-beam interaction and therefore would severely degrade the performance of circular colliders. To avoid this issue, we will consider a more traditional correction system, as illustrated in Fig. 7, right next to the final telescope.

This kind of scheme was successfully demonstrated in a final focus test beam [18] as a single-pass system, achieving $73 \mathrm{~nm}$ beam size in the vertical plane. The chromatic correction section shown in Fig. 7 consists of four $90^{\circ} / 90^{\circ}$ cells, which makes a unit transformation. Four identical dipoles are introduced to generate dispersions at the 


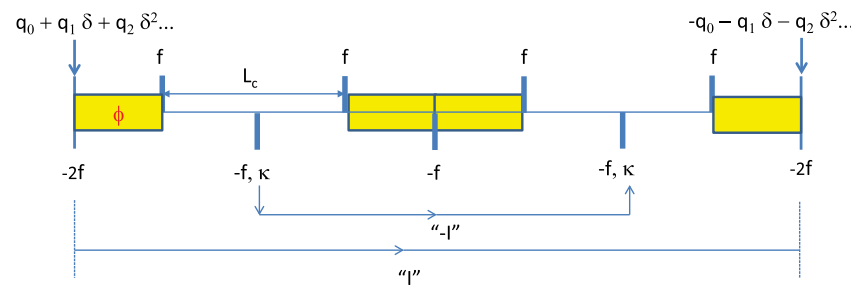

FIG. 7. A schematic layout of a chromatic correction module in the vertical plane. The focusing length $f=L_{c} / 2 \sqrt{2}$ for $90^{\circ}$ cells.

positions of the sextupoles and they are paired with $180^{\circ}$ phase difference to cancel the dispersion at the end of the module. The sextupoles with strength $\kappa$ are paired also in "-I" so that their geometric aberrations are canceled. This pair of sextupoles are placed in $180^{\circ}$ phase difference relative to the final doublet for the first-order chromaticity correction.

Using the transfer maps of the elements, $\nu_{y}(\delta)$ can be computed analytically [9]. At the first-order of $\delta$, we find
$\nu_{y}^{\prime}=-\frac{L_{c}\left[16(2+\sqrt{2})+(5+3 \sqrt{2}) L_{c}^{2} \kappa \phi\right]+4 M^{2} L^{*}}{8 \pi M^{2} \beta_{y}^{*}}$,

where $\phi$ is the bending angle of the dipole and $L_{c}$ the cell length. For simplicity, we have used the simplified map in Eq. (9) for the final telescope. Setting $\nu_{y}^{\prime}=0$, we derive the strength of the sextupoles,

$$
\kappa=-\frac{16(2+\sqrt{2}) L_{c}+4 M^{2} L^{*}}{(5+3 \sqrt{2}) L_{c}^{3} \phi} .
$$

Similarly, the linear chromatic compensation can be carried out numerically for the final focus system shown in Fig. 8. It consists of the final telescope we introduced earlier in Fig. 5 and the chromatic correction illustrated in Fig. 7. Here we show the $\mathrm{W}$ functions after the first-order correction in Fig. 9.

Setting the sextupole to the value in Eq. (14), we compute the phase advance up to the next order of $\delta$ and find indeed that the first-order chromaticity has vanished and the second-order,

$$
\nu_{y}^{\prime \prime}=\frac{10(146+103 \sqrt{2}) L_{c}-(17+13 \sqrt{2}) M^{2} L^{*}-(43+30 \sqrt{2}) q_{0}\left(M^{2} L^{*}\right)^{2}}{2 \pi(43+30 \sqrt{2}) M^{2} \beta_{y}^{*}} .
$$

For a vanishing second-order chromaticity, $\nu_{y}^{\prime \prime}=0$, we need

$q_{0}=\frac{10(146+103 \sqrt{2}) L_{c}-(17+13 \sqrt{2}) M^{2} L^{*}}{(43+30 \sqrt{2})\left(M^{2} L^{*}\right)^{2}}$.
Note that the quadrupole strength is very small if $M \gg 1$. For thick quadrupoles, this scheme can be implemented simply by shortening the half quadrupole at the beginning while lengthening the other half at the end. Since the unit transformation is retained, there is no need to rematch the lattice.

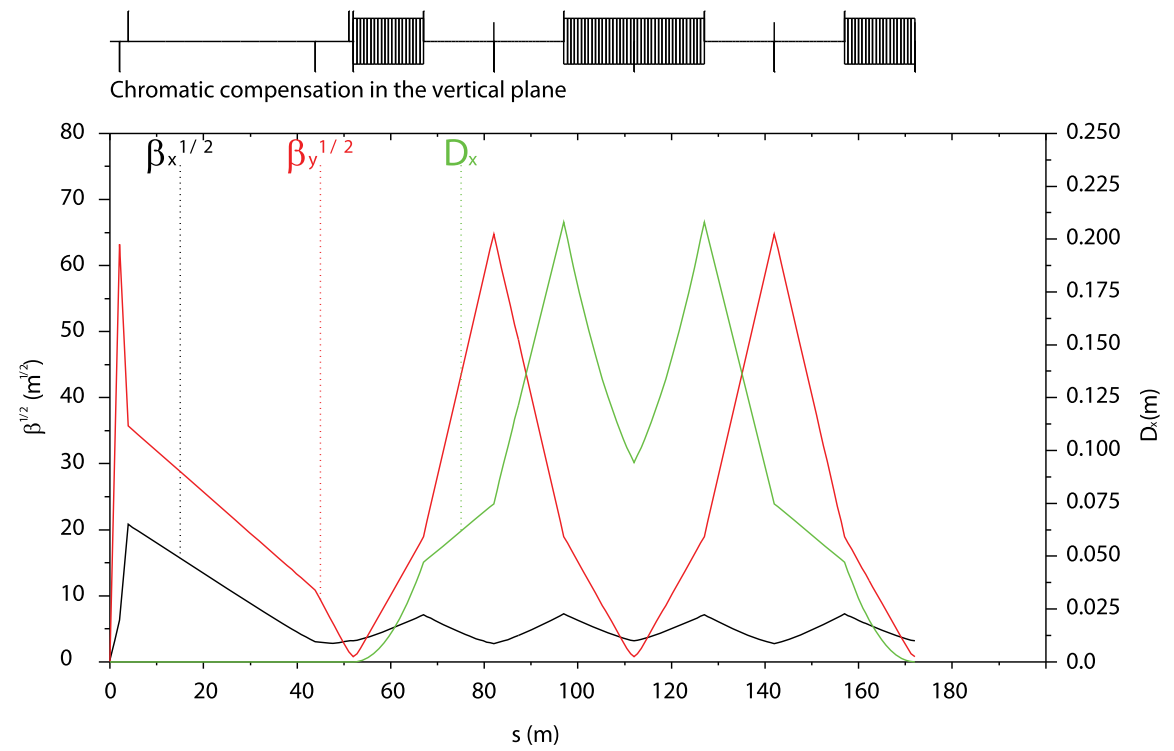

FIG. 8. Lattice functions in a final focusing system with local chromatic compensation section. The parameters in the chromatic correction module are $L_{c}=30 \mathrm{~m}$ and $\phi=\pi / 500$. 


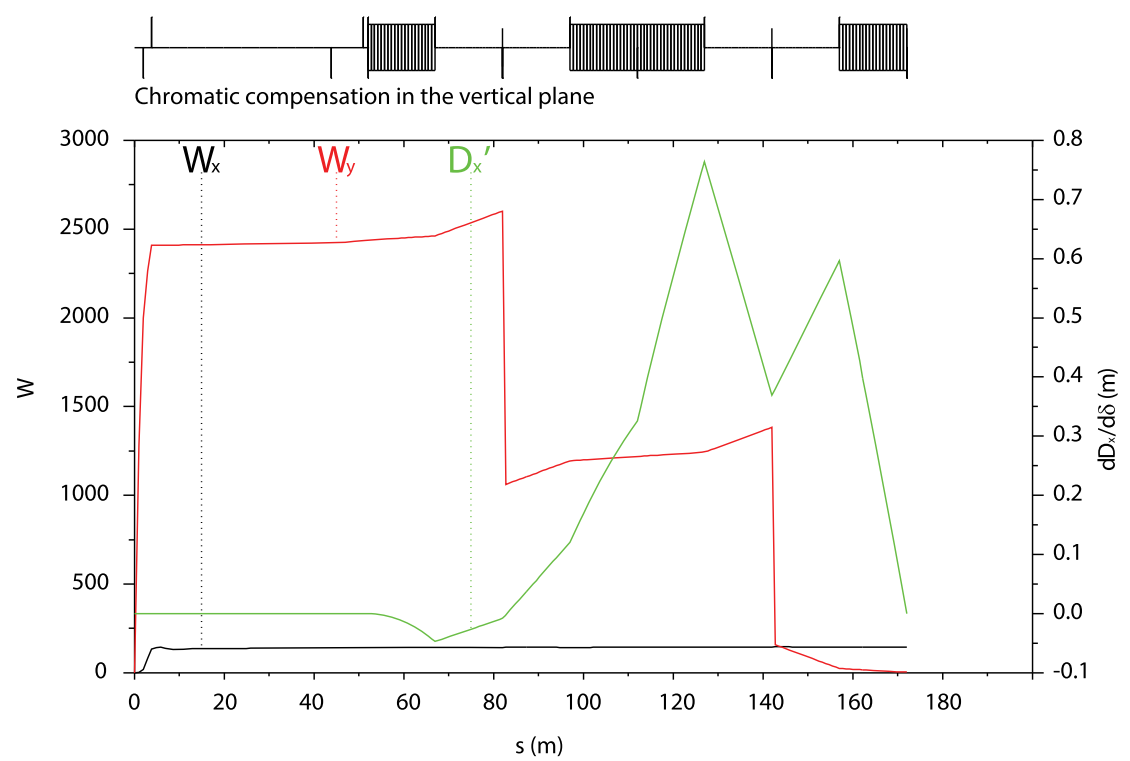

FIG. 9. W function and the second-order dispersion in the final focusing system with the local chromatic compensation.

Continuing this procedure to the third order, we have

$$
\begin{aligned}
\nu_{y}^{\prime \prime \prime}= & -\frac{1}{4 \pi(3649+2580 \sqrt{2}) L_{c} M^{4} L^{*} \beta_{y}^{*}} \\
& \times\left\{400(21267+15038 \sqrt{2}) L_{c}^{3}\right. \\
& +8(43023+30424 \sqrt{2}) L_{c}^{2} M^{2} L^{*} \\
& -8(859+605 \sqrt{2}) L_{c}\left(M^{2} L^{*}\right)^{2}+[12458+8809 \sqrt{2} \\
& \left.\left.+2(3649+2580 \sqrt{2}) q_{1} L_{c}\right]\left(M^{2} L^{*}\right)^{3}\right\} .
\end{aligned}
$$

To correct this term, we simply set

$$
\begin{aligned}
q_{1}= & -\frac{1}{2(3649+2580 \sqrt{2}) L_{c}\left(M^{2} L^{*}\right)^{3}} \\
& \times\left[400(21267+15038 \sqrt{2}) L_{c}^{3}\right. \\
& +8(43023+30424 \sqrt{2}) L_{c}^{2} M^{2} L^{*} \\
& -8(859+605 \sqrt{2}) L_{c}\left(M^{2} L^{*}\right)^{2} \\
& \left.+(12458+8809 \sqrt{2})\left(M^{2} L^{*}\right)^{3}\right] .
\end{aligned}
$$

In practice, this term can by generated by a sextupole along with a dipole in the final telescope. This kind of sextupole is commonly called the Brinkmann sextupole [19].

Note that these long expressions of the nonlinear chromaticity are obtained with the help of MATHEMATICA [20]. This procedure can be continued to higher orders but becomes quite messy. For higher order chromaticity, the result can be summarized by a simple pattern,

$$
\begin{aligned}
\nu_{y}^{\prime \prime} & =A_{0} q_{0}+B_{0}, \\
\nu_{y}^{\prime \prime \prime} & =A_{0} q_{1}+B_{1}, \\
& =\cdots, \\
\nu_{y}^{(n)} & =A_{0} q_{n-2}+B_{n-2},
\end{aligned}
$$

where

$$
\begin{aligned}
A_{0}= & -\frac{L_{c}^{2}}{32 \pi M^{2} \beta_{y}^{*}}\left[512(3+\sqrt{2})+32(16+11 \sqrt{2}) L_{c}^{2} \kappa \phi\right. \\
& \left.+(43+30 \sqrt{2}) L_{c}^{4} \kappa^{2} \phi^{2}\right], \\
B_{0}= & \frac{L_{c}}{8 \pi M^{2} \beta_{y}^{*}}\left[80+56 \sqrt{2}+(1+2 \sqrt{2}) L_{c}^{2} \kappa \phi\right] .
\end{aligned}
$$

Most importantly, $B_{i}$ does not have dependence on $q_{j}$ for $j \leq i$ so that the high chromaticity can be zeroed out order by order by solving the linear equations in Eq. (19). It is worth noting that the quadratic term of the sextupole strength $\kappa$ in the expression of $A_{0}$ is rather large. As a result, $q_{i}$ tends to be small and therefore they are very efficient correctors.

It is well known that the second-order chromaticity can be corrected by a small phase trombone between the final doublet and the sextupole pair. Here we simply use a thinlens quadrupole at the imaging point to achieve the same effect. The high-order pattern naturally follows in the Lie algebra approach.

Similarly, the order-by-order procedure of chromatic compensation can be carried out numerically. The results up to the third-order chromaticity correction are shown in Fig. 10. Clearly, the bandwidth becomes large as the correcting order increases. 

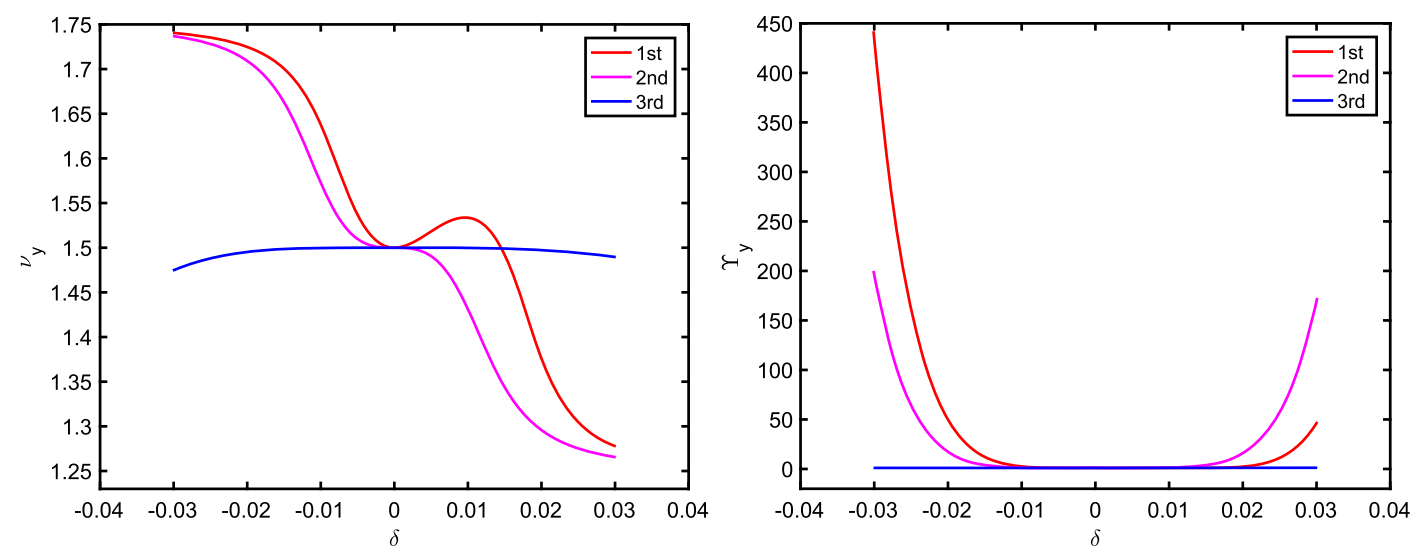

FIG. 10. Chromatic bandwidth of the final focus system with different order of corrections shown on (left) the vertical phase advance in unit of $2 \pi$ and (right) the Sands' mismatch parameter $\Upsilon$.
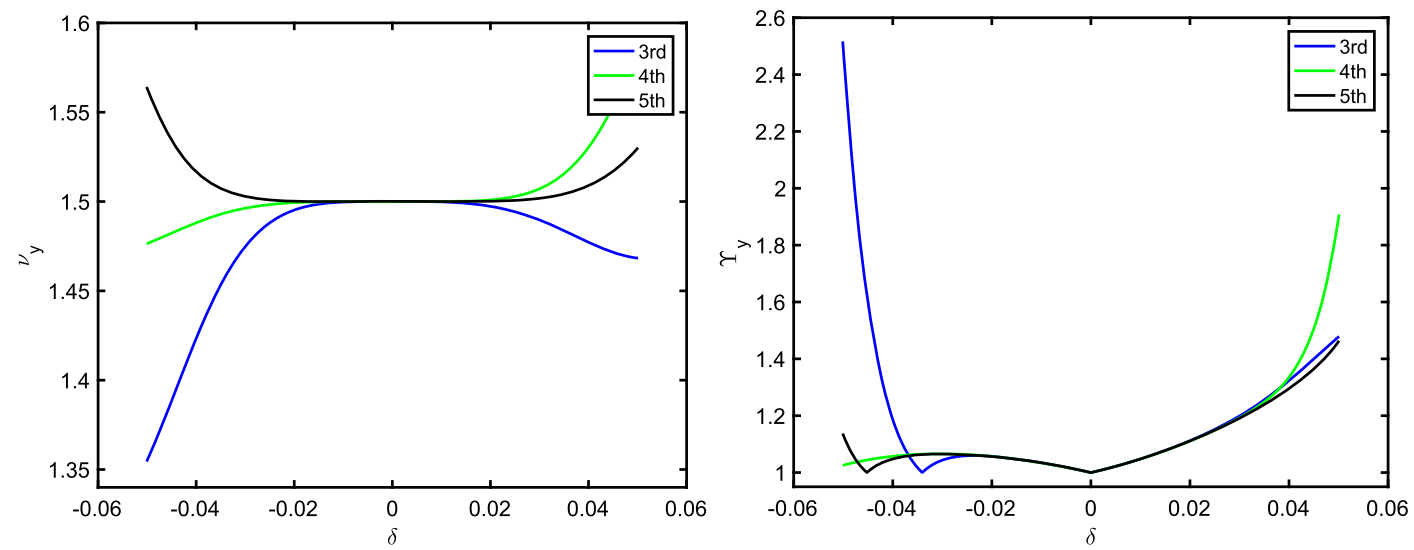

FIG. 11. Improved chromatic bandwidth of the final focus system with higher order of corrections shown on (left) the vertical phase advance in unit of $2 \pi$ and (right) the Sands' mismatch parameter $\Upsilon$.

For $\pm 2 \%$ of the momentum bandwidth, it is sufficient to correct up to the third order of $\delta$. The figure shows that the third-order compensation is essential for a large bandwidth.

To double the bandwidth to $\pm 4 \%$, we have to correct up to the fifth order of the momentum deviation $\delta$ as shown in Fig. 11. In implementation, we need to use octupole and decapole magnets at the imaging points.

The horizontal chromaticity can be corrected by a similar scheme. Its chromatic correction module can be constructed by changing the sign of $f$ and $\phi$ in Fig. 7. If we add it right after the vertical correction section, then there will be another set of linear equations similar to Eqs. (13) and (19). In general, there are small cross talks between the horizontal and vertical planes. So we need to solve for two unknowns for two linear equations at each order of $\delta$.

\section{FINAL FOCUS SYSTEM}

It is always challenging to design a final focus system (FFS) in a circular collider. In the CHF, it becomes even more so because of a smaller $\beta_{y}^{*}(1 \mathrm{~mm})$ and a longer distance $L^{*}(2$ meter) required to achieve a factory-level luminosity of $10^{34} \mathrm{~cm}^{-2} \mathrm{~s}^{-1}$.

Using the principles outlined in the previous sections, we have designed a practical optics of the FFS shown in Fig. 12. The FFS starts with a final transformer (FT), continues with a chromatic correction in the vertical (CCY) and then the horizontal plane (CCX), and ends with a simple matching section. The FFS has two secondary imaging points and fits in a 321-meter section.

The FT contains four quadrupoles, including the final focusing doublet. The betatron phase advances are $180^{\circ}$ in both planes. A dipole is added to the section to generate the dispersion at the end, where the sextupole can be used to correct the third-order chromaticity in the vertical plane. As a result, the dipoles in the other regions are rearranged slightly to retain the dispersion within the FFS as shown in Fig. 12.

The CCY consists of four $90^{\circ}$ FODO cells and makes a unit of betatron transformer. The module starts at the middle of the defocusing quadrupole to enhance the peak of the vertical beta function at the positions of a pair of 

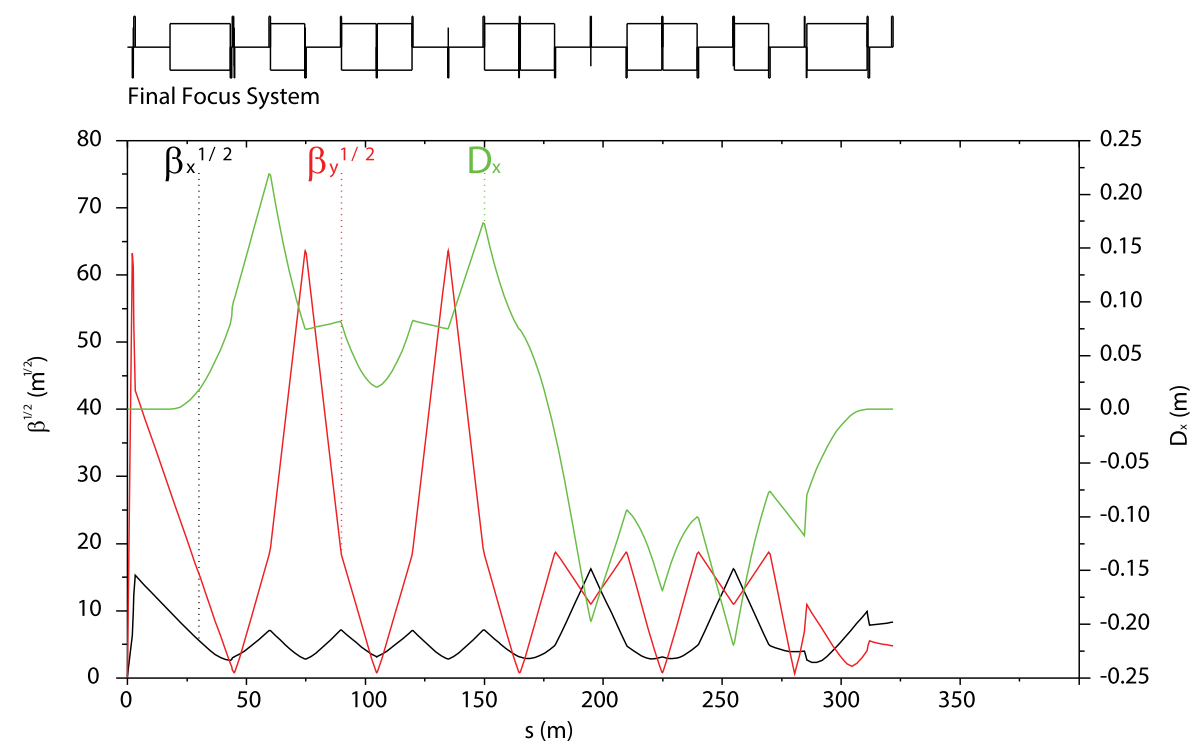

FIG. 12. Lattice functions in a final focusing system with local chromatic compensation section.

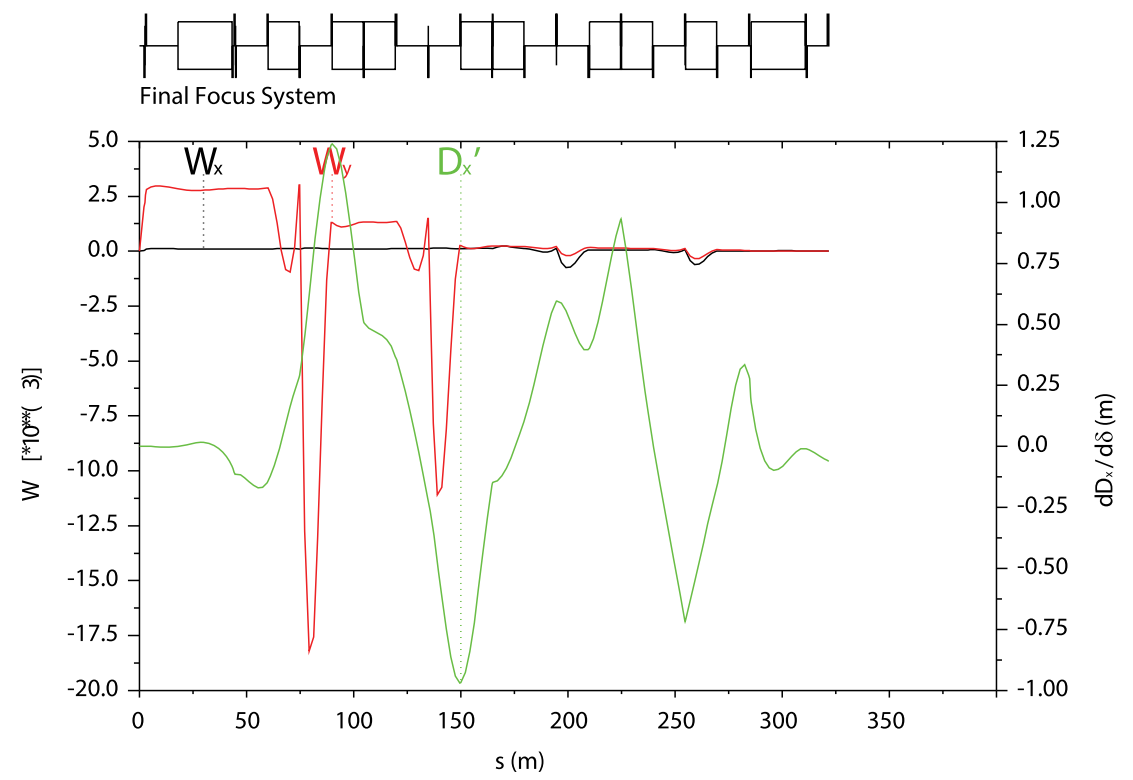

FIG. 13. W function and the second-order dispersion in the final focusing system with the local chromatic compensation.

sextupoles separated by -I transformation. Five dipoles with an equal bending angle provide dispersions at the locations of the sextupoles. At the end of the CCY, we have the second imaging point at which the lattice functions are identical to those at the first one.

Similarly, we construct the CCX, but starting at the middle of the focusing quadrupole. There are five dipoles that generate the dispersion with negative bending angles. The amplitudes of the angles are chosen to be the same as those in the CCY so that there is no net bending from the FFS. At the end of the CCX, we have a section matching to the optics of the dispersion suppressor.

The nonlinear chromatic effects can be characterized by the high order derivatives of the lattice functions. These derivatives can be computed [9] using the technique of the differential algebra [21]. For the FFS, we start with the initial condition, $\beta_{x}=0.1 \mathrm{~m}$ and $\beta_{y}=1 \mathrm{~mm}$, and calculate the lattice functions and their derivatives element by element down to the end.

TABLE II. The chromatic tune shifts from the FFS after the correction.

\begin{tabular}{lc}
\hline \hline Derivatives of tunes & Values \\
\hline$\partial \nu_{x, y} / \partial \delta$ & $-0.6,-0.7$ \\
$\partial^{2} \nu_{x, y} / \partial \delta^{2}$ & $+5,+11$ \\
$\partial^{3} \nu_{x, y} / \partial \delta^{3}$ & $-1494,+514$ \\
\hline \hline
\end{tabular}



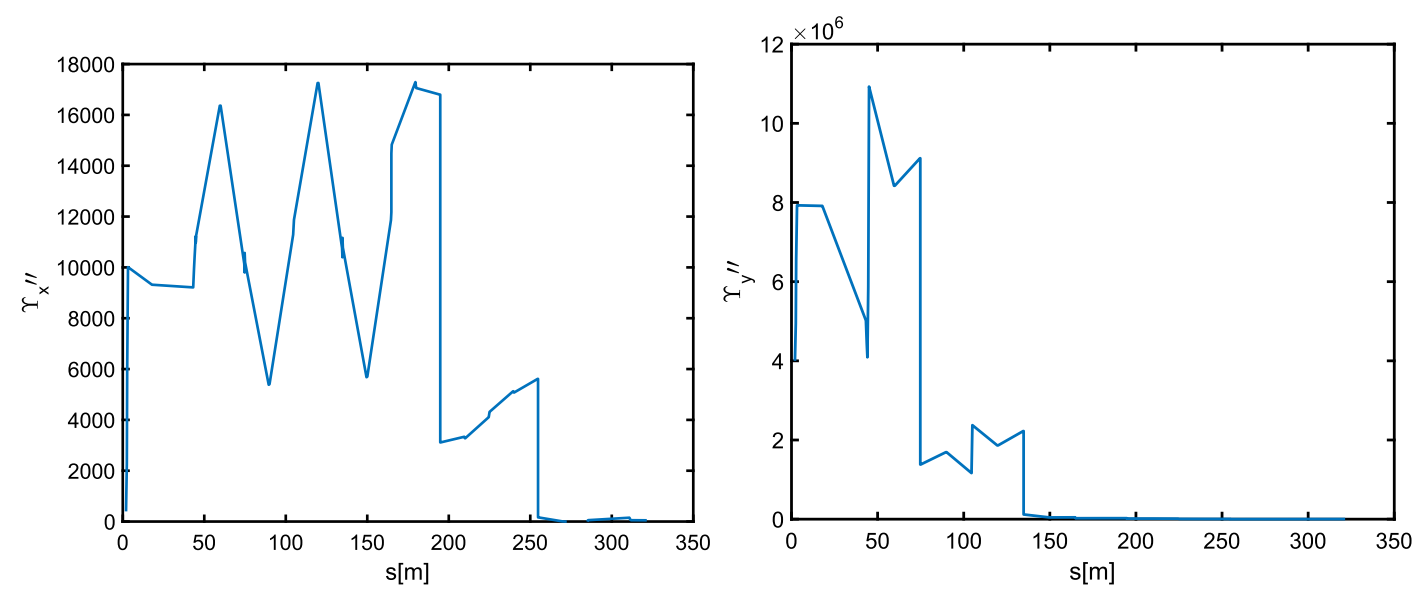

FIG. 14. The second derivative of the Sands' mismatch parameter $\Upsilon$ as a function of $s$ in the horizontal (left) and vertical (right) planes.

The first order chromaticity is compensated by two pairs of sextupoles in the CCY and CCX respectively in the horizontal and vertical planes; the second-order ones by slight changes of betatron phases between the final doublet and the sextupole pairs; and finally the third-order ones by the two sextupoles at the two secondary imaging points where the beta functions are at the minimum. We compensate the chromaticities locally up to the end of CCX. As we have mentioned previously, at every order, we simply solve two coupled linear equations. The results of the chromatic compensation are summarized in Table II. Small values in the table are due to the matching section. In addition, we plot the $\mathrm{W}$ functions and the second-order dispersion in Fig. 13. It is worth noting that there is a leak of the second-order dispersion out of the region but it is very small.

To show how the higher order chromaticity is compensated, we plot the second derivative of the mismatch parameter $\Upsilon$ as a function of the distance away from the IP in Fig. 14. It shows where the large corrections occur, namely at the vertical steps, and also that the system is quite orthogonal between the horizontal and vertical planes.

\section{COLLIDER}

Using $60^{\circ}$ FODO cells in the arc, we build a collider lattice with two interaction regions. Since the lattice has a twofold symmetry, half of the betatron tunes are slightly above the half integer, which enhances the dynamic focusing from the beam-beam interaction.

The main parameters of the collider are summarized in Table III. The beam energy and luminosity are essentially determined or required by the high-energy physics for the study of Higgs. The circumference was chosen to be close to the value in CEPC [22] so that the design can be useful. Since the loss of synchrotron radiation is so large at this high energy, the beam current is limited by the rf power nearly $200 \mathrm{MW}$ for both beams. Finally, the lattice functions at the interaction point are mostly determined by the required luminosity. In addition, the parameters of the FFS in the collider are the same as these in the final focus system sections, in particular $L^{*}=2$ meter and $\beta_{x}^{*}=0.1$ meter.

The global linear chromaticities are corrected by the two families of sextupoles in the arcs without tweaking the sextupoles in the interaction regions. The results of chromatic corrections are tabulated in Table IV. The third-order terms become rather large largely due to the nonlinear mismatch of the chromatic optics between the interaction region and the arcs. Further improvement is possible with

TABLE III. Main parameters of a circular Higgs factory.

\begin{tabular}{lc}
\hline \hline Parameter & Value \\
\hline Energy, $E_{0}[\mathrm{GeV}]$ & 125 \\
Luminosity/IR, $\mathcal{L}\left[10^{34} \mathrm{~cm}^{-2} \mathrm{~s}^{-1}\right]$ & 2.55 \\
Circumference, $\mathcal{C}[\mathrm{km}]$ & 52.7 \\
Beam curent, $I[\mathrm{~mA}]$ & 13 \\
SR power, $P_{S R}[\mathrm{MW}]$ & 50 \\
Beta function at IP, $\beta_{y}^{*}[\mathrm{~mm}]$ & 1 \\
Beam-beam parameter, $\xi_{y}$ & 0.1 \\
Natural emittance, $\epsilon_{x}[\mathrm{~nm}]$ & 4.5 \\
Bunch length, $\sigma_{z}[\mathrm{~mm}]$ & 1.85 \\
Energy loss per turn, $U_{0}[\mathrm{GeV}]$ & 3.85 \\
rf voltage, $V_{r f}[\mathrm{GV}]$ & 8.45 \\
rf frequency, $f_{r f}[\mathrm{MHz}]$ & 650.0 \\
Tune, $\nu_{x}, \nu_{y}, \nu_{z}$ & $225.04,227.14,0.165$ \\
Damping time, $\tau_{x}, \tau_{y}, \tau_{z}[\mathrm{~ms}]$ & $11.4,11.4,5.7$ \\
\hline \hline
\end{tabular}

TABLE IV. The chromatic tune shifts in the collider that contain two interaction regions.

\begin{tabular}{lc}
\hline \hline Derivatives of tunes & Values \\
\hline$\partial \nu_{x, y} / \partial \delta$ & 0,0 \\
$\partial^{2} \nu_{x, y} / \partial \delta^{2}$ & $-167,+790$ \\
$\partial^{3} \nu_{x, y} / \partial \delta^{3}$ & $+27978,-19146$ \\
\hline \hline
\end{tabular}




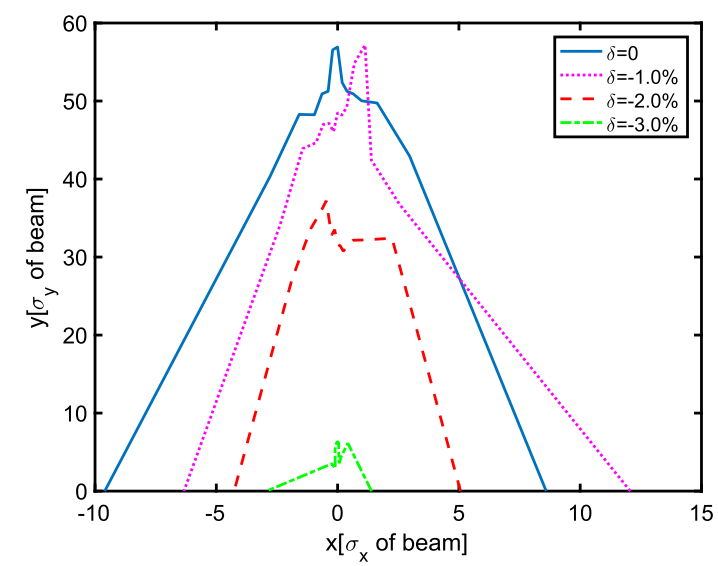

FIG. 15. Dynamic aperture of the collider with various momentum deviations.

numerical optimizations $[23,24]$ but is not investigated given the analytical nature of this paper.

Aside from the chromatic compensation, we need to mitigate the geometric-chromatic aberrations in the FFS to achieve a good momentum aperture in the collider. We find that the largest aberration is generated by the pairs of the sextupoles at the peaks of the beta functions because of the chromatic error in -I between two sextupoles. Fortunately, the aberration can be corrected by a pair of decapoles at the same places as the sextupole pairs.

Finally, we evaluate the dynamic aperture of the collider by tracking the particles with various momentums. The tracking is carried out with synchrotron oscillation and the radiation damping. The orbit and optics errors due to the sawtooth energy profile are corrected by tapering the settings for all magnetic elements. As shown in Fig. 15, though the degradation of the off-momentum aperture is large, there is sufficient momentum acceptance to retain the particles in the long tail distribution of energy due to beamstrahlung. Most importantly, there is large enough dynamic aperture in the vertical plane to accommodate the large tail generated by the beam-beam interaction.

Here, we have developed an analytical method to effectively compensate nonlinear chromaticity in the final focusing system. We have achieved $2 \%$ momentum acceptance in a lattice with an ultralow beta interaction region. Six families of sextupoles are sufficient in the chromatic correction. As shown in this paper, such optics in a collider with a consistent set of accelerator parameters, especially with a large momentum aperture, has been demonstrated in design.

\section{DISCUSSION}

We have developed an analytical method to compensate the chromaticity order by order in momentum deviation $\delta$ in the conventional FFS. The variables for the correction are nearly orthogonal and their impacts are clearly identified.
Moreover, the formulas of their settings lead to many insights of how to optimize the system. These settings could also be a good starting point for further numerical optimization [25] with more variables, especially in the arcs, similar to a more elaborate numerical optimization [26].

\section{ACKNOWLEDGMENTS}

I would like to thank Alexander Chao, Katsunobu Oide, Richard Talman, and Frank Zimmermman for many stimulating and helpful discussions. This work was supported by the Department of Energy under Contract No. DE-AC0276 SF00515.

[1] PEP-II design group, Report No. SLAC-418, 1993.

[2] KEKB accelerator group, Report No. KEK-Report-95-7, 1995.

[3] M. Benedikt and F. Zimmermann, Outline and status for the FCC-ee design study, ICFA Beam Dynamics Newsletter 67, 86 (2015).

[4] CEPC-SPPC Study Group, Reports No. IHEP-CEPC-DR2015-01 and No. IHEP-AC-2015-01, 2015.

[5] V. I. Telnov, Restriction on the Energy and Luminosity of $e^{+} e^{-}$Storage Rings due to Beamstrahlung, Phys. Rev. Lett. 110, 114801 (2013).

[6] B. Richter, Very high electron-positron colliding beams for the study of weak interactions, Nucl. Instrum. Methods 136, 47 (1976).

[7] K. L. Brown, Report No. SLAC-PUB-4159, 1987.

[8] R. D. Ruth, Single Particle Dynamics in Circular Accelerator, edited by M. Month and M. Dienes, AIP Proc. No. 153 (AIP, New York, 1985), Vol. 1, p. 166.

[9] Y. Cai, Symplectic maps and chromatic optics in particle accelerators, Nucl. Instrum. Methods Phys. Res., Sect. A 797, 172 (2015).

[10] D. Shatilov, Y. Alexahin, V. Lebedev, and A. Valishev, LIFETRAC code for the weak-strong simulation of the beam-beam effects in Tevatron, in Proceedings of the 21st Particle Accelerator Conference, Knoxville, 2005 (IEEE, Piscataway, NJ, 2005), TPAT084.

[11] K. Ohmi, Simulation of beam-beam effects in a circular $e^{+} e^{-}$collider, Phys. Rev. E 62, 7287 (2000).

[12] E. D. Courant and H. S. Snyder, Theory of the alternatinggradient synchrotron, Ann. Phys. (N.Y.) 3, 1 (1958).

[13] M. G. Minty and F. Zimmermann, Measurement and Control of Charged Particle Beams (Spinger-Verlag, Berlin, 2003).

[14] M. Sands, SLAC Internal Report No. SLAC-AP-85, 1991.

[15] B. W. Montague, Linear optics for improved chromaticity correction, CERN LEP Collider Note 165, 1979.

[16] A. Bogomyagkov and E. Levichev, Momentum acceptance optimization in FCC-ee lattice, in Proceedings of IPAC'16, Busan, Korea, 2016 (JAcoW, Geneva, 2016), THPOR19.

[17] P. Raimondi and A. Seryi, Novel Final Focus Design for Future Linear Colliders, Phys. Rev. Lett. 86, 3779 (2001).

[18] V. Balakin et al., Focusing of Submicron Beam for TeV-Scale $e^{+} e^{-}$Linear Colliders, Phys. Rev. Lett. 74, 2479 (1995). 
[19] R. Brinkmann, DESY Report No. M-90-14, 1990.

[20] Mathematica version 9, A system for doing mathematics by computer, Wolfram Research Inc., 2014.

[21] M. Berz, Differential algebra description of beam dynamics to very high order, Part. Accel. 24, 109 (1989).

[22] D. Wang et al., CEPC parameter choice and partial double ring design, in Proceedings of IPAC'16, Busan, Korea, 2016 (JAcoW, Geneva, 2016), THPOR10.

[23] R. Tomas, Nonlinear optimization of beam lines, Phys. Rev. ST Accel. Beams 9, 081001 (2006).
[24] H. Garcia-Morales and R. Tomas, Final-focus systems for multi-TeV linear colliders, Phys. Rev. ST Accel. Beams 17, 101001 (2014).

[25] Y. Wang et al., Dynamic aperture study of the CEPC main ring with interaction region, in Proceedings of IPAC'16, Busan, Korea, 2016 (JAcoW, Geneva, 2016), THPOR012.

[26] K. Oide et al., Design of beam optics for the FCC-ee collider rings, Phys. Rev. Accel. Beams (to be published). 